\title{
Implications of Lifecourse Epidemiology for Research on Determinants of Adult Disease
}

\author{
Sze Liu, ${ }^{1}$ \\ Richard N. Jones, ${ }^{2}$ \\ M. Maria Glymour ${ }^{1}$
}

\begin{abstract}
Many diseases commonly associated with aging are now thought to have social and physiologic antecedents in early life. Understanding how the timing of exposure to early life risk factors influences later-life health may illuminate mechanisms driving adult health inequalities and identify possible points for effective interventions. Recognizing chronic diseases as developing across the lifecourse also has implications for the conduct of research on adult risk factors for disease. We review alternative conceptual models that describe how the timing of risk factor exposure relates to the development of disease. We propose some expansions of lifecourse models to improve their relevance for research on adult chronic disease, using the relationship between education and adult cognitive decline and dementia as an example. We discuss the important implications each of the lifecourse conceptual models has on study design, analysis, and interpretation of research on aging and chronic diseases. We summarize several research considerations implied by the lifecourse framework, including: advantages of analyzing change in function rather than onset of impairment; the pervasive challenge of survivor bias; the importance of controlling for possible confounding by early life conditions; and the likely heterogeneity in responses of adults to treatment.
\end{abstract}

Key words: Lifecourse epidemiology, aging, chronic disease, models, dementia

Recommended Citation: Liu S, Jones RN, Glymour MM. Implications of Lifecourse Epidemiology for Research on Determinants of Adult Disease. Public Health Reviews. 2010;32:489-511.

\footnotetext{
${ }^{1}$ Department of Society, Human Development, and Health, Harvard School of Public Health.

${ }^{2}$ Division of Gerontology, Department of Medicine, Beth Israel Deaconess Medical Center, Institute for Aging Research, Hebrew SeniorLife, Harvard Medical School.

Corresponding Author Contact Information: M. Maria Glymour at mglymour@hsph. harvard.edu; Department of Society, Human Development, and Health, Harvard School of Public Health, Boston, MA 02115.
} 


\section{INTRODUCTION}

Accumulating evidence from lifecourse epidemiology indicates that many diseases typically diagnosed in adulthood have social and physiologic antecedents much earlier in life..$^{1-3}$ Although the research faces many analytic challenges, the fundamental premise - illnesses of middle and late life are often shaped by developmental processes experienced in utero, in childhood, in adolescence or early adulthood - has strong empirical support. ${ }^{4-6}$ This result has several important implications for appropriate design, analysis, and interpretation of research on aging and chronic diseases. In many cases, an understanding of the role of early life conditions is relevant even for substantive research questions relating to exposures or outcomes exclusively encountered in adults. Research on adult chronic diseases focuses on identifying opportunities to intervene to improve population health, whether via clinical treatments, behavioral interventions, or policy changes. Understanding the lifecourse antecedents of diseases associated with aging can provide essential insight into selecting the timing and structure of interventions in order to successfully improve population health. ${ }^{7}$ Lifecourse epidemiology can both highlight new or unrecognized opportunities for improving population health and inform methods to test long-standing hypotheses about the determinants of adult disease. These models are also valuable for understanding the likely population health trends in a world undergoing a rapid demographic transformation. In some cases, lifecourse epidemiology suggests the stage was set for major population health changes decades ago, during the early lives of current cohorts of adults.

In this paper, we summarize the alternative etiologic models in lifecourse epidemiology, discuss limitations in the current literature, and propose some expansions of lifecourse models to improve their relevance for research on adult chronic disease. These models are important because paradigms for the development of adult disease shape the research questions we ask and the methods used to address such questions. Applying a life course perspective challenges researchers to consider the dynamic process and multidimensional nature of health and well-being in adulthood. After reviewing examples of such conceptual representations, we discuss several implications of these models for research on determinants of adult disease: the value of analyzing change in function rather than onset of impairment whenever possible; the pervasive challenge of survivor bias; the importance of controlling for possible confounding by early life conditions; and the likely heterogeneity in responses of adults to treatment.

For clarity, we illustrate our discussion with the example of the effects of education and cognitive stimulation on adult cognitive decline and dementia. 
We consider possible benefits of education to be mediated by immersion in a cognitively stimulating environment, characterized by novelty and intellectual challenges, and assume that an individual may have such experiences at any time in life, via work or leisure activities, even if not participating in formal education. The possible effects of both educational attainment and adult cognitive engagement on dementia and cognitive decline are active research areas. $^{8-11}$ The importance of lifecourse models for understanding cognitive aging and dementia has recently received increased attention. ${ }^{12-15}$ However, many of the ideas are immediately relevant to other outcomes, such as disability.

\section{LIFECOURSE EPIDEMIOLOGY FRAMEWORKS}

a.

Childhood

Education

Adult Cognitive

Stimulation

$\longrightarrow$ Dementia

b.

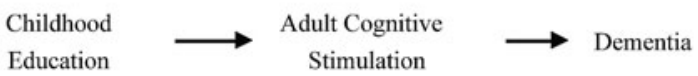

c.

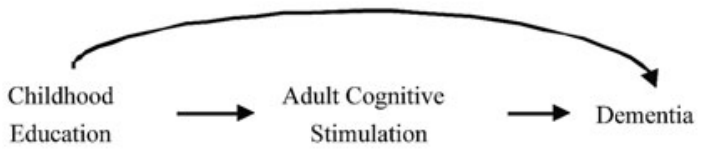

d.

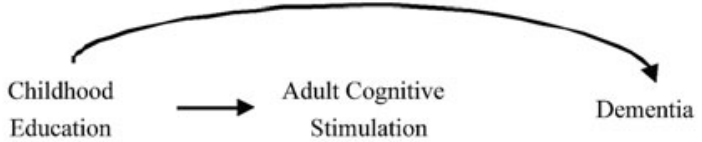

e.

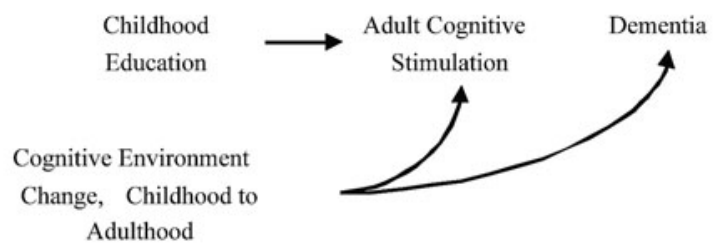

Fig. 1. Alternative lifecourse models linking the timing of exposure to stimulating cognitive environment and dementia risk. (a) Immediate risk; (b) Social trajectory; (c) Cumulative risk; (d) Early life latency; (e) Social mobility effects. 
Lifecourse epidemiology takes as a principle that, although many diseases are primarily diagnosed in old age, such conditions may nonetheless reflect damage (or benefits) incurred from exposures much earlier in life. Although the exact characterizations of alternative lifecourse models differ slightly across the published literature, researchers distinguish among competing models linking the timing of exposure to a potential risk factor to the manifestation of disease (Figure 1a-e).,16 We use "risk factor" here to indicate an exposure or treatment that affects health, not merely a marker for such an exposure. For example we focus on exposures that would increase risk of disease for an individual who was exposed to the risk factor compared to the risk the individual would have had if he or she had not been exposed. ${ }^{17}$ The distinction between such a causal risk factor and proxies or markers for causal risk factors is that causal risk factors suggest opportunities for interventions to improve health, whereas changing markers or proxies for causal agents is not anticipated to provide health benefits.

Immediate effects models posit brief etiologic periods so that exposure to a causal risk factor increases risk of disease quite promptly. ${ }^{18}$ In other words, it assumes individuals experience a decrease in disease risk concurrently or shortly after the risk factor is removed. For example, Figure 1a represents the assumption that low levels of cognitive stimulation in midlife immediately affect dementia risk, and educational or cognitive experiences in childhood or early adulthood are irrelevant. In this model, childhood education has no effect on dementia, either directly or mediated through adult cognitive experiences. Interventions to enrich the cognitive environments of children would not be expected to have any effect in this model, but late life interventions would be expected to be beneficial.

Many exposures, particularly those related to social position follow a "sticky" trajectory, where childhood conditions shape adult exposures. For these exposures, social trajectory models (Figure 1b) are more appropriate than immediate risk models. For example, high-quality childhood education may enable someone to obtain a challenging and engaging job, whereas poor childhood education may lead to monotonous work. In this case, if adult cognitive engagement affects dementia risk (as in the immediate risk model) then childhood education will also affect dementia risk via its influence on adult cognitive engagement. In Figure 1b, there is no direct effect of childhood exposure on adult outcomes, it is only the adult exposure that directly harms (or in this case, benefits) adult health. These cascading models are sometimes called "chain of risk" or "risk of risk" models. This model suggests there may be several options for designing effective interventions, because intervention in either childhood or adulthood could 
be beneficial. To identify the most promising opportunities it is helpful to consider possible "elbow" or "lever" periods, when the intervention would have the largest effects on the exposure. Such "elbows" occur when the direction of future exposure trajectory is most malleable; possible turning points might occur during the first encounter with formal schooling, at the moment of transition from school to work, or at retirement.

In contrast to social trajectory models, which assume that early life social factors do not "get under the skin" but only increase risk of future damage, cumulative biological models posit that each period of risk factor exposure induces permanent physiologic harm (Figure 1c). This damage accumulates over the lifecourse, increasing risk of later illness with each additional exposure period. Even if the risk is removed in adulthood, the individual will still experience the physiologic damage accumulated prior to removal. Cumulative biological models often seem the most plausible description of risk-factor-health associations. Allostatic load models are premised on the accumulation of physical damage over the lifecourse..$^{19,20}$ Cumulative models may be especially apt for social risk factors. There are often many possible pathways via which social risk factors affect health, and different mechanisms may be relevant at different points in the pathway. ${ }^{21}$ However, physical toxins such as lead may also have cumulative biological effects on adult cognitive outcomes. For some diseases, including dementia, the diagnosis of the disease typically occurs long after the initial physiologic damage begins. The diagnosis is the tail end of a long, accumulating pathologic process. The cumulative biological model can also incorporate the concept of historically linked lives and intergenerational transfer of risk. Previous studies have suggested parental health and health behaviors have direct and early effects on their children, placing them at greater risk for subsequent detrimental health outcomes. ${ }^{22,23}$

For some exposures, there may be an especially sensitive window of time during which the exposure is extremely influential, and after the temporal window closes, the exposure is no longer relevant. Such sensitive period or latency models typically invoke a key developmental period. For example, periods of rapid tissue growth, development of aspects of neurologic "architectural" structure, ${ }^{24-27}$ or plasticity in epigenetic marks ${ }^{28,29}$ imply that environmental encounters during these periods will be unusually influential. ${ }^{30,31}$ A familiar example of a sensitive period is language development: humans are especially responsive to language exposure during early life..$^{32}$ If there is no exposure during the sensitive age, full fluency is rarely achieved. Immigration studies suggest that many behavioral factors are developmentally sensitive as well. ${ }^{33}$ For example, the patterns of smoking among immigrant children depends on the age of immigration: early 
immigrants are more likely to adopt the smoking patterns of the receiving culture (e.g., high smoking rates among American teenagers), while those who immigrate as adults are more likely to retain the smoking patterns of their country of origin. ${ }^{34,35}$ There is also evidence of possible early life critical period effects for specific adult physiological features. For example, adult obesity is associated with intrauterine environment and rapid weight gain in infancy. ${ }^{36,37}$

The final category of model, which we call a mobility effects model, explicitly addresses change as an exposure in and of itself (Figure 1e). For example, one may hypothesize that children successfully adapt to their early life conditions but those adaptations may be harmful if they encounter a different environment in adulthood (e.g., nutritional environment). Research on socioeconomic status sometimes posits that social mobility whether up or down - is itself very stressful and results in worse health. ${ }^{38}$

\section{CHOOSING AMONG THESE MODELS}

For specific combinations of exposures and outcomes, evidence on which of the lifecourse models is most appropriate is drawn from both theoretical considerations and empirical analyses. The models have divergent implications regarding the statistical associations between early and midlife exposures and the outcome. The statistical implications of each model can be read off the diagrams and thus used to empirically assess the plausibility of each model. ${ }^{12}$ For example, an implication of the latency model in Figure $1 \mathrm{~d}$ is that midlife cognitive stimulation should have no relationship to probability of dementia after controlling for childhood educational exposures. In other words, in the model:

Probability $($ dementia $)=c_{0}+c_{1} * X_{1}+c_{2} * X_{2}$ the coefficient $c_{2}$ should be null, while the coefficient $c_{1}$ should predict dementia. If both exposures were measured perfectly and there were no confounders of either the childhood or the adult risk factor, this finding would rule out the immediate risk, social trajectory, or cumulative risk models. Alternatively, if we found that both childhood and midlife exposures were independently predictive of the outcome, this would be inconsistent with the immediate risk, social trajectory, or latency models and instead suggest that a cumulative effects model characterized the relationship between the risk factor and the outcome.

Unfortunately, the assumptions under which this approach succeeds are very stringent and rarely completely credible. Available measures of childhood conditions are invariably inadequate and many studies also have 
imperfect measures of the adult risk factor ${ }^{39}$ Further, if childhood conditions influence adult conditions (as in the social trajectory model) then these analyses entail all of the challenges of direct/indirecteffects decomposition. ${ }^{38-40}$ Finally, testing effects of mobility versus effects of beginning or final position is challenging because of the colinearity between any two of these, given the third. For example, adult socioeconomic status is exactly determined by childhood socioeconomic status plus change in socioeconomic status from childhood to adulthood. ${ }^{38}$ Convincing solutions have not been developed, but advances may come from substantive assumptions about possible mediators of effects; and or about the functional relationship between any of the three and the outcome. ${ }^{41-43}$

\section{EXPANDING THESE FRAMEWORKS TO DISTINGUISH DEVELOPMENT FROM DISEASE}

Many important health conditions, including dementia and disability, reflect the intersection of developmental processes, pathological or disease processes, and recovery or resilience. These three components may have distinct antecedents. The criteria for clinical diagnoses of Alzheimer's disease include a threshold component based on deficits in specific cognitive domains. ${ }^{44}$ Although most diagnostic criteria include consideration of whether the deficit represents a decline from prior functioning, ceiling effects in currently used measurement scales make it difficult to identify deficits in very high performing individuals. As a result, deficits are intrinsically related to performance below a threshold.

Whether or not a person performs below a cognitive threshold may be influenced by attained cognitive skills (e.g., IQ), a pathologic process (e.g., ischemia induced neural damage or deposition of amyloid plaques) in the brain, or cognitive plasticity or resilience that enables maintenance of function even after an individual incurs neurologic damage. For example, Figure 2a contrasts two very different hypothetical lifecourse cognitive trajectories. For both individuals, cognitive performance meets the criteria for dementia at the same age. However, one individual experienced a longer period of cognitive development in early life, and then a much faster rate of decline, perhaps due to a more severe or aggressive type of dementia. The other individual ceased enhancement of cognitive skills relatively early in life, but then experienced a slow rate of cognitive decline during old age. Modeling age of dementia diagnosis (or risk of dementia diagnosis) does not fully distinguish between these two distinct pathways. Similarly, in Figure $2 b$, one cognitive trajectory reflects a series of mild strokes, each 
causing modest cognitive decrements, with essentially no post-stroke cognitive recovery. The other trajectory reflects a series of severe strokes, each followed by substantial cognitive recovery. These two individuals meet the threshold for dementia at roughly the same time, but the factors that influenced the severity of stroke versus stroke recovery are probably quite different.
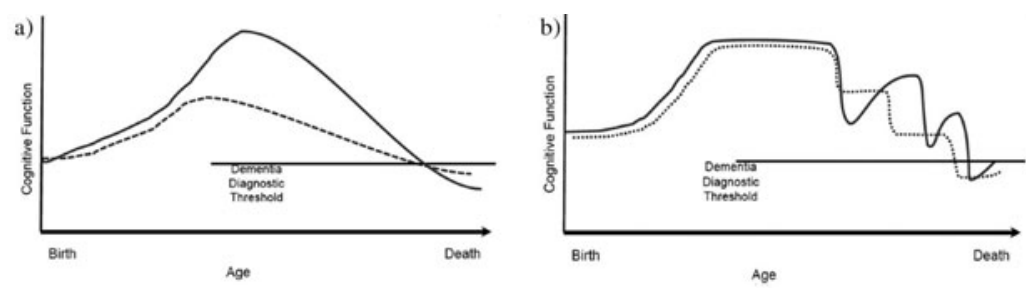

Fig. 2. Alternative cognitive trajectories across the lifecourse, resulting in meeting threshold criteria at the same age. (a) Longer period of cognitive development but faster rate of age-related decline. (b) More severe cognitive decrements after stroke, but greater neurologic recovery.

Conversion to a clinically recognized dementia is an important outcome to the extent that this is the point where individuals are likely to lose independence, require greater services, and place greater burden on caregivers. Such considerations lead clinicians naturally to focus research on the endpoint of dementia diagnosis. However, defining diagnosis as the outcome of interest forces unnecessary and unrealistic assumptions about the nature of the disease under study. Dementia is a spectrum disorder, and the identification of cases involves applying arbitrary thresholds to a continuous process. ${ }^{45}$ Both the major underlying pathologies that are understood to cause dementia and cognitive decline, and the behavioral manifestation of those pathologies, are exhibited quantitatively. ${ }^{46}$ Even individuals with low cognitive scores within the "normal" range are at elevated risk of mortality and institutionalization, ${ }^{47,48}$ suggesting the underlying biological process of cognitive decline may best be characterized as a continuous - instead of a threshold - marker of risk. Breaking a continuous outcome into discrete categories through the application of arbitrary thresholds, even if clinically well-informed, is often bad statistical practice ${ }^{49}$ Moreover, conflation of the outcome in this way is likely to create unnecessary barriers to identifying exposures that influence either cognitive development or the disease process that results in decline. This is especially problematic in the case of dementia, because normal variability 
in cognitive test performance among adults is much larger than variability in the rate of decline or disease development.

Longitudinal study designs combined with analyses examining changes in indicators of disease or functional outcomes are a better option. Lifecourse models can reflect the distinct influences of developmental processes, disease processes, and recovery/resilience. These models explicitly represent the hypothesized causal mechanism linking any specific risk factor to dementia. Often, early life factors are likely to influence late life disease because of their effects on development, and therefore vulnerability to physiologic insults encountered later in life. Figure 3 shows one possible model, in which early life conditions directly influence neurologic development and also the development of certain types of neurologic disease, but do not directly affect recovery/resilience.

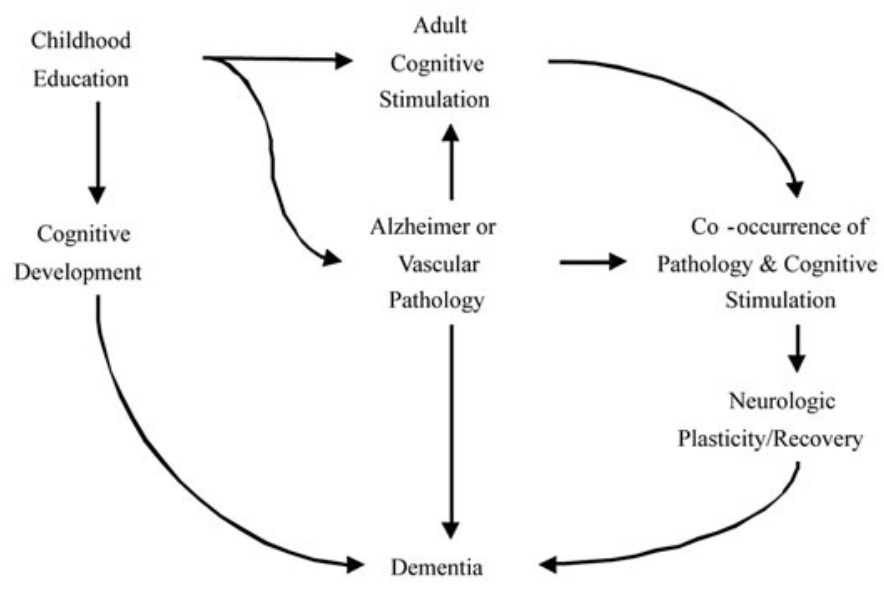

Fig. 3. Lifecourse model distinguishing between developmental, pathological, and recovery processes linking education or cognitive engagement to dementia.

In this hypothetical model, adult cognitive stimulation influences recovery after neurologic injury, but has no direct effect on either early life neurologic development or the risk of developing pathologies that harm cognitive function (e.g., Alzheimer's or cerebrovascular disease). This model shows the co-occurrence of stimulation and pathology as a single variable on the pathway between cognitive stimulation and cognitive recovery. This is to represent the (testable) hypothesis that cognitive stimulation is only important in the context of injury or disease.$^{50}$ This diagram also illustrates a 
serious potential bias in lifecourse research: early indicators of disease may influence exposure to risk factors, for example, if small cognitive impairments in early stages of dementia lead individuals to withdraw from cognitively challenging activities. These expanded models can be valuable for research on many types of complex health conditions because they express and illustrate how to empirically test hypothesized causal mechanisms by which lifecourse risk factors affect the outcome. We now turn to discussing how recognition of lifecourse factors can inform research on adult risk factors and adult disease.

\section{POTENTIAL FOR CONFOUNDING BY EARLY LIFE CONDITIONS}

Confounding bias arises when there is a common cause of the exposure and outcome variables. ${ }^{51-53}$ For example, in Figure 1d, we would say that childhood education confounds the association between adult cognitive stimulation and dementia, because childhood education influences both, but the effect of education is not mediated by adult cognitive stimulation. Early life conditions are possible confounders in chronic disease research, because childhood exposures are often strong predictors of adult risk factors, such as socioeconomic position (SEP), diet and other behaviors, ${ }^{54-56}$ and cardiovascular and immune function, ${ }^{4,57-59}$ all factors believed to be important in determining cognitive performance level in later life. In most studies, childhood conditions are either measured poorly or not measured at all. If one were attempting to test a social trajectory versus cumulative effects model, for example, the adequacy of measurement of early and late life exposures is critical. Often early life exposures are measured with relatively more error than late life exposures. Thus, parameter estimates for early life exposures will be biased towards the null, leading to a risk of spuriously rejecting the cumulative effects model in favor of the social trajectory model. The proliferation of longitudinal studies with multiple measures of the same construct (e.g., SEP) across time may help address the problem. ${ }^{35,60}$ However, even with operational measures of early-life conditions, fully eliminating confounding bias depends on accurately modeling the cascade of "upstream" lifecourse exposures that influence adult risk factors and health outcomes. ${ }^{30}$

\section{THE IMPORTANCE OF MODELING CHANGE INSTEAD OF LEVEL}

As suggested by the discussion above and Figure 2, the findings of lifecourse epidemiology imply that if we are interested in identifying 
determinants of the pathological process (e.g., plaque deposition), the appropriate outcomes are markers of the pathological process. Exclusive use of cognitive performance at any given age, or even "onset" of disease as a surrogate outcome is a weak design because there are many influences other than pathology that contribute to variability in performance. Rather, to study the development of pathologies related to aging, it is important to focus on indicators of the accumulation of pathology. This is often impractical, and therefore studies of changes in performance across time may be a suitable endpoint. Although disease onset may appear to intrinsically correspond with a change in function, this is often misleading. Many diseases have roots years, decades, or even generations prior to the date of diagnosis or even the earliest clinical symptoms.

A leading hypothesized mechanism linking education and dementia is that education may reduce risk of manifesting cognitive impairment in the face of a given level of neuropathology. ${ }^{59,60}$ Education probably affects neurologic development ${ }^{61,62}$ and cognitive skills may likewise influence attained education. But this association tells us little about the etiology of, for example, amyloid plaques. In research on the development of agerelated pathology, the effects of education on early life cognitive function may simply be "noise." However, the developmental effects of education are likely to be huge, ${ }^{61,63-65}$ so this noise is a serious impediment to research.

In a community population of middle-aged or "young-old" individuals, the cognitive performance differences associated with an attained year of education are sometimes several times as large as the cognitive performance differences associated with a chronological year of age (calculations available from the authors). Age-related change becomes more important among older samples, but even in quite aged populations, a great deal of the cross-sectional variance in cognitive function is attributable to stable personal characteristics such as education.

Focusing on cognitive decline or change may also help avoid conflation of cohort differences with aging effects. Conceptually, a birth cohort represents a unique social category. The life chances of individuals within a birth cohort will be affected by intra-cohort characteristics (e.g., demographic composition of the cohort), inter-cohort characteristics (e.g., relative size compared to other generations). Longitudinal studies have reported significant cohort differences in baseline cognitive performance, such that successive generations appear to have better cognitive test performance, differences which are not fully attributable to schooling. ${ }^{66-68}$ In cross-sectional designs, this pattern will tend to overstate the effect of aging because older individuals differ from younger individuals in both age and birth cohort. It is necessary to examine rate of change to understand the 
effects of aging per se. Although there may be differences in rate of cognitive decline across cohorts, evidence for this is much more mixed. ${ }^{67,69,70}$

Cognitive decline may also be a better predictor of subsequent physical outcomes than cognitive level, due to the improved capacity to capture change over time..$^{71,72}$ Given the dynamic nature of cognitive functioning, there may be substantial measurement errors in cognitive function measured cross-sectionally. Cognitive trajectories may be a more accurate characterization of the ongoing process of cognitive aging. Conversely, maintenance of cognitive function, even relatively low but stable function, may be reflective of a successful cognitive aging trajectory. With thresholdbased outcomes such as onset of dementia, healthy individuals with low but largely stable cognitive function may be categorized as demented with only a small decrement in performance due to a transient factor.

Insufficient analytic distinctions between determinants of development and determinants of disease may have contributed to the longstanding debate regarding the effects of education on dementia and cognitive aging. A first generation of studies found that education predicted higher baseline cognition levels, reduced risk of cognitive impairment (performance below a threshold), and reduced risk of dementia in the elderly ${ }^{73-84}$ Results from a number of early studies suggested that education slowed the rate of cognitive decline ${ }^{85}$ However, these results were challenged by several subsequent reports using improved longitudinal methods, and the overall association is now in question. ${ }^{8,9,86-89}$ Change-point models that flexibly model rate of decline prior to the formal diagnosis of dementia indicate that the association between education and level of function might be in the opposite direction as the association between education and rate of change. ${ }^{90}$

Despite the above discussion, in some cases, level of functioning is more substantively important than rate of change. For example, projections of disease burden and assessments of individual clinical needs may need to incorporate information on diagnoses and other criteria based on level of performance. ${ }^{91}$

\section{LIFELONG ACCUMULATION OF RISK CONTRIBUTES TO SELECTION AND SURVIVOR BIAS IN STUDIES OF AGING}

Studies of early life determinants of adult health are potentially subject to severe bias from selective survival: the early life adversity may influence the chance of surviving to old age, so the only elderly who experienced extreme childhood adversity are those with some other protective factor. ${ }^{39}$ In extreme forms this phenomena has been called "reverse epidemiology". ${ }^{92}$ This bias can also apply to adult risk factors, and is thought to account for 
the mixed findings regarding the association between cigarette smoking and dementia among older adults. This association generally attenuates in older samples, presumably because the lethality of smoking results in highly winnowed populations of very old smokers. ${ }^{93}$ In the example of education and cognitive function, education promotes survival, and thus low-education elderly may have other unobserved, beneficial personal traits. If these traits influence health, they would bias any estimate of the association between education and health among the survivors. Because of the association between education and cognitive engagement, the same bias may apply to estimates of the association between adult cognitive activities and health. Such selection bias can be addressed analytically using common missing data methods such as inverse probability weighting to account for competing risks if the other factors are measured..$^{94,95} \mathrm{In}$ many instances, it has been shown that although this bias is a theoretical possibility, it is not severe unless survival is quite low (e.g., in very old samples). Studies of individuals above age 80 or 90 , however, may face severe bias due to selective survival.

\section{HETEROGENEOUS EXPOSURE EFFECTS FOR DETERMINANTS OF ADULT HEALTH}

The vast majority of biomedical research focuses on the effects of treatment on the population average health (either overall average or within specified population subgroups). However, there is no reason to assume that exposure has the same effect on everyone in a population, or even everyone within a pre-specified subgroup. ${ }^{96}$ There is growing recognition that focusing exclusively on average effects may be misleading and inappropriate. ${ }^{97,98} \mathrm{We}$ do not expect medical interventions to affect all individuals similarly: we take it for granted that medical treatments are targeted to those individuals most likely to benefit (e.g., anti-hypertensive medications are given to individuals with hypertension, not normotensive individuals). The field of personalized genetics is premised on the hope that there are many heretofore unmeasured genetic characteristics that determine treatment responsiveness.

Extending this idea to social conditions, we can see that unmeasured social risk factors may also modify the effects of exposure for any type of adult risk factor. Early life adversity may exert an influence on health and well-being throughout life so that individuals who arrive at old age as "low performers" may already have experienced a very distinct life history of adverse social conditions prior to the initiation of a study. These exposures may modify the response of the individual to new exposures or treatments. 
As a result, when considering any adult risk factor, the effects on "high performers" may be very different than the effects on "low performers". For example, aging may result in faster cognitive decline among "low performers" who are already at risk in late middle-age, and have less severe affects on "high performers" who arrive at late middle-age with a lifelong cumulative advantage. Modeling this heterogeneity can be challenging. For example, our ability to detect differences in the pace of cognitive change over time requires cognitive outcome measures with interval level scaling properties. Brief mental status tests and single domain assessments rarely satisfy such strict requirements. If such measures were available, we would anticipate an increasing variability in cognitive function across age, as in Figure 4.

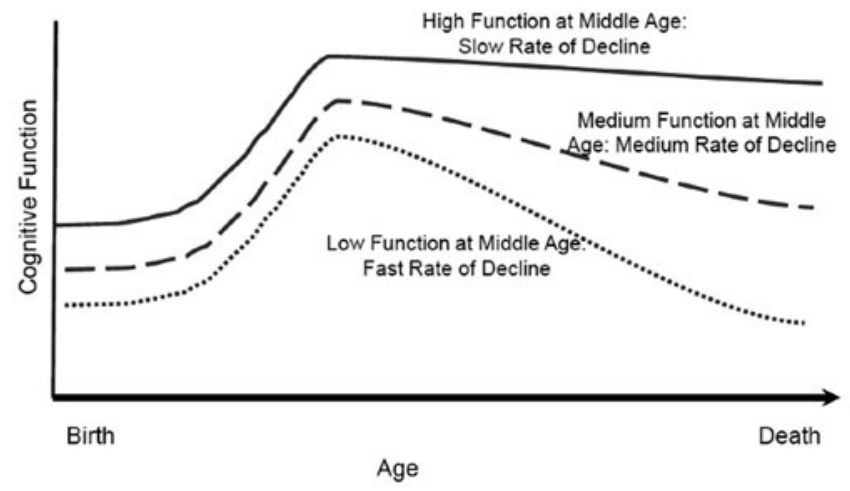

Fig. 4. Individuals who experience advantage across life and arrive at middle age with high cognitive function may experience cognitive decline more slowly than those who encounter disadvantage across life. If so, this will result in greater increasing variability in cognitive function across age.

The standard approach to examine possible variation in exposure effects is to include an interaction between the exposure and observed covariates (e.g., low socioeconomic status as a child) in the regression model. At the very least, current studies using interaction terms to assess heterogeneity in average exposure effects should conduct and report subgroup analyses (with appropriate account for multiple testing). ${ }^{99}$ However, treatment responsiveness may differ across unmeasured or unknown personal traits. There are alternative methods that can explicitly model heterogeneous treatment effects on high and low baseline performers, without explicitly specifying interaction models. Quantile regression models can be used to describe the effect of the risk factor on the entire distribution of the outcome, 
rather than just the mean as in conventional ordinary least squares regression models. One study using quantile regression found variations in the association between early life influences and adult body mass index (BMI). Among smaller women, birth weight was strongly associated with adult BMI while maternal weight gain was strongly associated with adult BMI among larger women. ${ }^{100}$ Arguably, individuals at the extreme end of the cognitive distribution are of greater concern for population health than individuals at the mean. Furthermore, rate of change for any given percentile may be dependent on that percentile. To date, no studies have considered how cognitive trajectories might differ for those at different parts of the distribution. Since continuous measures are generally more sensitive to group differences than categorical outcomes, quantile methods may also better capture differences in associations between various risk factors and continuous measure of cognition.

\section{CONCLUSIONS AND FUTURE RESEARCH DIRECTIONS}

A large body of evidence now indicates that many adult chronic diseases are shaped by early life exposures. Although the importance of a lifecourse approach to understanding chronic disease is increasingly recognized, public health interventions applying these principles have been limited. When designing interventions, it is valuable to clearly articulate the lifecourse model assumed to link the exposure and the outcome, and identify lifecourse periods when the exposure can be changed. If there is a single early life sensitive period for a specific exposure-outcome combination, it may be useless to initiate interventions later in life. If harm accumulates slowly throughout life, interventions to change exposure in adulthood may take years before they show benefits. Explicit recognition of lifecourse models can help improve design and effectiveness of future intervention programs to promote healthy aging. ${ }^{7}$ Public health interventions informed by a lifecourse perspective have focused on time points presumed to have special plasticity, such as early childhood. Researchers have advocated that maternal and child health interventions using a lifecourse approach include: 1) information strategies that use age-appropriate messages and venues to disseminate health information across the lifespan; 2) administrative strategies that link health services across the life span; 3 ) organizational strategies that reconfigure programs to reflect integrated health goals and 4) environmental strategies that address community building and environmental exposure. ${ }^{101-104}$ Early Childhood Comprehensive Systems enacted by several US states already incorporate a lifecourse 
perspective and use such a multi-pronged approach. ${ }^{105}$ Recent developments in trials of pharmaceutical treatments for Alzheimer's disease, such as amyloid immunotherapy, suggest that intervening early may be essential. Treatments may be most effective at interrupting the neuropathological process long before conventional diagnostic criteria are met, although the optimal timing is unknown. ${ }^{106}$ The public health challenge is to improve our understanding of the lifecourse processes linking modifiable exposures and major diseases and use this information to inform effective interventions across the lifespan. Although preventive interventions early in life may have larger benefits than comparable interventions later in the lifecourse for some outcomes, most adult health outcomes presumably reflect an ongoing interplay between biological, environmental and social factors. Emerging evidence suggests physiologic and functional plasticity in many domains persists throughout life. ${ }^{107-109}$ Interventions targeting time points in mid or late adulthood may clearly have substantial benefits.

Articulating and applying theoretical models of lifecourse processes (e.g., immediate risk, social trajectory, cumulative effects, latency, or mobility) to longitudinal data can help clarify interpretation and comparison of regression results. Even when the focus of research is late life risk factors, it is important to consider the role of lifecourse processes in generating the observed associations between the risk factor and the outcome; creating barriers to intervention efforts to remediate the risk factor; or modifying the responsiveness of individuals to the risk factor. Explicit theorizing about lifecourse processes will help inform better intervention design, even when the intervention is for older adults. ${ }^{7}$ Finally, lifecourse models are important as we project into the future the health of an aging population and consider priorities for current health investments. In coming years, we may experience either substantial health benefits or health costs due to investments made during the early lives of aging cohorts. Likewise, investments made now in generations of children or younger adults may have health consequences for decades into the future.

Acknowledgments: The authors gratefully acknowledge financial support from the W.K. Kellogg Foundation Kellogg Health Scholars Program (P0117943) and the Milton Fund for Harvard University faculty (MMG).

Conflicts of Interest: None declared.

\section{REFERENCES}

1. Barker DJ, Martyn C. The maternal and fetal origins of cardiovascular disease. J Epidemiol Community Health. 1992;46:8-11. 
2. Kuh D, Ben-Shlomo Y, (editors). A lifecourse approach to chronic disease epidemiology: Tracing the origins of ill-health from early to adult life. Oxford (UK): Oxford University Press; 1997.

3. Power C, Hertzman C. Social and biological pathways linking early life and adult disease. Br Med Bull. 1997;53:210-22.

4. Galobardes B, Smith GD, Lynch JW. Systematic review of the influence of childhood socioeconomic circumstances on risk for cardiovascular disease in adulthood. Ann Epidemiol. 2006;16:91-104.

5. Galobardes B, Lynch JW, Smith GD. Childhood socioeconomic circumstances and cause-specific mortality in adulthood: Systematic review and interpretation. Epidemiol Rev. 2004;26:7-21.

6. Pollitt RA, Rose KM, Kaufman JS. Evaluating the evidence for models of life course socioeconomic factors and cardiovascular outcomes: a systematic review. BMC Public Health. 2005;5:7.

7. Berkman LF. Social epidemiology: Social determinants of health in the United States: Are we losing ground? Ann Rev Public Health. 2009;30:27-41.

8. Wilson RS, Hebert LE, Scherr PA, et al. Educational attainment and cognitive decline in old age. Neurology. 2009;72:460.

9. Alley D, Suthers K, Crimmins E. Education and cognitive decline in older Americans: Results from the AHEAD sample. Res Aging. 2007;29:73-94.

10. Karlamangla AS, Miller-Martinez D, Aneshensel CS, Seeman TE, Wight RG, Chodosh J. Trajectories of cognitive function in late life in the United States: Demographic and socioeconomic predictors. Am J Epidemiol. 2009;170:331-42.

11. Stern C, Munn Z. Cognitive leisure activities and their role in preventing dementia: a systematic review. Int J Evid Based Healthc. 2010;8:2-17.

12. Whalley LJ, Dick FD, McNeill G. A life-course approach to the aetiology of late-onset dementias. Lancet Neurol. 2006;5:87-96.

13. Glymour MM, Manly JJ. Lifecourse social conditions and racial and ethnic patterns of cognitive aging. Neuropsychol Rev. 2008;18:223-54.

14. Singh-Manoux A, Richards M, Marmot M. Socioeconomic position across the lifecourse: How does it relate to cognitive function in mid-life? Ann Epidemiol. 2005;15:572-8.

15. Turrell G, Lynch JW, Kaplan GA, Everson SA, Helkala EL, Kauhanen J, Salonen JT. Socioeconomic position across the lifecourse and cognitive function in late middle age. J Gerontology B Psychol Sci Soc Sci. 2002;57:S43-51.

16. Wadsworth M. Health inequalities in the life course perspective. Soc Sci Med. 1997;44:859-69.

17. Pearl J. Causality. Cambridge (UK): Cambridge University Press; 2000.

18. Kraemer H, Kazdin A, Offord D, Kessler RC, Jensen PS, Kupfer DJ. Coming to terms with the terms of risk. Arch Gen Psychiatry. 1997;54:337.

19. McEwen BS. Stress, adaptation, and disease: Allostasis and allostatic load. In: McCann SM, Lipton JM, (editors). Annals of the New York Academy of Sciences, Vol 840: Neuroimmunomodulation: Molecular aspects, integrative systems, and clinical advances. New York (NY): New York Academy of Sciences; 1998. p.33-44. 
20. Seeman T, Epel E, Grueewald T, Karlamangla A, McEwen BS. Socio-economic differentials in peripheral biology: Cumulative allostatic load. Ann N Y Acad Sci. 2010;1186:223-39.

21. Link BG, Phelan J. Social conditions as fundamental causes of disease. J Health Soc Behav. 1995;Spec:80-94.

22. Cowell AJ. The relationship between education and health behavior: some empirical evidence. Health Econ. 2006;15:125-46.

23. Gilman SE, Martin LT, Abrams DB, Kawachi I, Kubzansky L, Loucks EB, et al. Educational attainment and cigarette smoking: a causal association? Int $\mathrm{J}$ Epidemiol. 2008;37:615-24.

24. Barker DJ. The fetal and infant origins of adult disease. BMJ. 1990;301:1111.

25. Barker DJP, Lackland DT. Prenatal influences on stroke mortality in England and Wales. Stroke. 2003;34:1598-602.

26. Fox S, Levitt P, Nelson III C. How the Timing and Quality of Early Experiences Influence the Development of Brain Architecture. Child Dev. 2010;81:28-40.

27. Gale CR, O'Callaghan FJ, Godfrey KM, Law CM, Martyn CN. Critical periods of brain growth and cognitive function in children. Brain. 2004;127:321-9.

28. Meaney MJ, Bhatnagar S, Larocque S, McCormick CM, Shanks N, Sharma S, et al. Early environment and the development of individual differences in the hypothalamic-pituitary-adrenal stress response. In: Pfeffer CR, (editor). Severe stress and mental disturbance in children. Washington (DC): American Psychiatric Press, Inc: 1996. p.85-127.

29. Meaney MJ, Aitken DH, Bodnoff SR, Iny LJ, Sapolsky RM. The effects of postnatal handling on the development of the glucocorticoid receptor systems and stress recovery in the rat. Prog Neuropsychopharmacol Biol Psychiatry. 1985;9:731-4.

30. Wang C. Beyond frequencies and coefficients - toward meaningful descriptions for life course epidemiology. Am J Epidemiol. 2006;164:122-5.

31. Pampel FC. The Persistence of Educational Disparities in Smoking. Soc Problems. 2009;56:526-42.

32. Doupe A, Kuhl P. Birdsong and human speech: Common themes and mechanisms. Ann Rev Neurosci. 1999;22:567-631.

33. Roshania R, Narayan K, Oza-Frank R. Age at arrival and risk of obesity among US immigrants. Obesity. 2008;16:2669-75.

34. Wilkinson A, Spitz M, Strom S, Prokhorov AV, Barcenas CH, Cao Y, et al. Effects of nativity, age at migration, and acculturation on smoking among adult Houston residents of Mexican descent. Am J Public Health. 2005;95:1043.

35. Mayer KU. New directions in life course research. Ann Rev Sociol. 2009;35: 413-33.

36. Robert CW, William HD. Role of the prenatal environment in the development of obesity. J Pediatr. 1998;132:768-76.

37. Stettler N, Kumanyika SK, Katz SH, Zemel BS, Stallings VA. Rapid weight gain during infancy and obesity in young adulthood in a cohort of African Americans. Am J Clin Nutr. 2003;77:1374-8. 
38. Hallqvist J, Lynch J, Bartley M, Lang T, Blane D. Can we disentangle life course processes of accumulation, critical period and social mobility? An analysis of disadvantaged socio-economic positions and myocardial infarction in the Stockholm Heart Epidemiology Program. Soc Sci Med. 2004;58:1555-62.

39. Glymour MM. Commentary: Selected samples and nebulous measures: Some methodological difficulties in life-course epidemiology. Int J Epidemiol. 2007;36:566-8.

40. Rosvall M, Chaix B, Lynch J, Lindström M, Merlo J. Similar support for three different life course socioeconomic models on predicting premature cardiovascular mortality and all-cause mortality. BMC Public Health. 2006;6:203.

41. Tu YK, West R, Ellison GTH, Gilthorpe MS. Why evidence for the fetal origins of adult disease might be a statistical artifact: The "reversal paradox" for the relation between birth weight and blood pressure in later life. Am J Epidemiol. 2005;161:27-32.

42. Tu Y, Woolston A, Baxter P, Gilthorpe MS. Assessing the impact of body size in childhood and adolescence on blood pressure: an application of partial least squares regression. Epidemiology. 2010;21:440.

43. Winship C, Harding D. A mechanism-based approach to the identification of age-period-cohort models. Soc Meth Res. 2008;36:362.

44. McKhann G, Drachman D, Folstein M, Katzman R, Price D, Stadlan EM. Clinical diagnosis of Alzheimer's disease: Report of the NINCDS-ADRDA Work Group* under the auspices of Department of Health and Human Services Task Force on Alzheimer's Disease. Neurology. 1984;34:939.

45. Hachinski V. Shifts in thinking about dementia. JAMA. 2008;300:2172-3.

46. Jorm A. A method for measuring dementia as a continuum in community surveys. In: Huppert FA, Brayne C, O'Connor DW, (editors). Dementia and normal aging. Cambridge (UK): Cambridge University Press; 1994. p.244-53.

47. McGuire LC, Ford ES, Ajani UA. Cognitive Functioning as a Predictor of Functional Disability in Later Life. Am J Geriatric Psych. 2006;14:36-42.

48. St John PD, Montgomery PR, Kristjansson B, McDowell I. Cognitive scores, even within the normal range, predict death and institutionalization. Age Ageing. 2002;31:373-8.

49. Altman DG. Categorizing continuous variables. John Wiley \& Sons, Ltd; 2005.

50. VanderWeele TJ, Robins JM. Directed acyclic graphs, sufficient causes, and the properties of conditioning on a common effect. Am JEpidemiol. 2007;166:1096.

51. Greenland S, Pearl J, Robins JM. Causal diagrams for epidemiologic research. Epidemiology. 1999;10:37-48.

52. Hernán MA, Hernandez-Diaz S, Robins JM. A structural approach to selection bias. Epidemiology. 2004;15:615-25.

53. Glymour MM, Greenland S. Causal Diagrams. In: Rothman KJ, Greenland S, Lash TL, (editors). Modern epidemiology. Philadelphia (PA): Lippincott Williams \& Wilkins; 2008. p.183-210. 
54. Van de Mheen H, Stronks K, Looman C, Mackenbach JP. Does childhood socioeconomic status influence adult health through behavioural factors? Int J Epidemiol. 1998;27:431.

55. Lynch J, Kaplan G, Salonen J. Why do poor people behave poorly? Variation in adult health behaviours and psychosocial characteristics by stages of the socioeconomic lifecourse. Soc Sci Med. 1997;44:809-19.

56. Melchior M, Moffitt T, Milne B, Poulton R, Caspi A. Why do children from socioeconomically disadvantaged families suffer from poor health when they reach adulthood? A life-course study. Am J Epidemiol. 2007;166:966-74.

57. Goodman E, McEwen B, Huang B, Dolan LM, Adler NE. Social inequalities in biomarkers of cardiovascular risk in adolescence. Psychosom Med. 2005;67:9.

58. Loucks E, Pilote L, Lynch J, et al. Life course socioeconomic position is associated with inflammatory markers: The Framingham Offspring Study. Social Science \& Medicine 2010.

59. Dufouil C, Alperovitch A, Tzourio C. Influence of education on the relationship between white matter lesions and cognition. Neurology. 2003;60:831-6.

60. Guralnik JM, Kritchevsky SB. Translating Research to Promote Healthy Aging: The Complementary Role of Longitudinal Studies and Clinical Trials. J Am Geriatr Soc. 2010;58:S337-42.

61. Ashenfelter O, Rouse C. Schooling, intelligence and income in America: Cracks in the bell curve. NBER Working Paper 1999;6902.

62. Glymour MM, Kawachi I, Jencks CS, Berkman LF. Does childhood schooling affect old age memory or mental status? Using state schooling laws as natural experiments. J Epidemiol Community Health. 2008;62:532-7.

63. Crone DA, Whitehurst GJ. Age and schooling effects on emergent literacy and early reading skills. J Educational Psychol. 1999;91:604-14.

64. Gorey KM. Early childhood education: A meta-analytic affirmation of the short- and long-term benefits of educational opportunity. School Psychol Q. 2001;16:9-30.

65. Darling-Hammond L. Cracks in the Bell Curve: How Education Matters. J Negro Education. 1995;64:340-53.

66. Finkel D, Reynolds CA, McArdle JJ, Pedersen NL. Cohort differences in trajectories of cognitive aging. J Gerontol B Psychol Sci Soc Sci. 2007;62:P286-94.

67. Karlamangla AS, Miller-Martinez D, Aneshensel CS, Seeman TE, Wight RG, Chodosh J. Trajectories of Cognitive Function in Late Life in the United States: Demographic and Socioeconomic Predictors. Am J Epidemiol. 2009; 170:331-42.

68. Schaie K. What can we learn from longitudinal studies of adult development? Res Human Develop. 2005;2:133-58.

69. Faul J. The effect of lifecourse socioeconomic position and health of trajectories of cognitive function in older adults. Ann Arbor (MI): University of Michigan; 2010.

70. Salthouse TA. When does age-related cognitive decline begin? Neurobiology Aging. 2009;30:507-14. 
71. Nikolova R, Demers L, Béland F. Trajectories of cognitive decline and functional status in the frail older adults. Arch Gerontol Geriatr. 2009;48:28-34.

72. Yaffe K, Lindquist K, Vittinghoff E, et al. The effect of maintaining cognition on risk of disability and death. J Am Geriatr Soc. 2010;58:889-94.

73. Rogers MAM, Plassman BL, Kabeto M, Barnes D, Simonsick EM, Newman A, et al. Parental Education and Late-life Dementia in the United States. J Ger Psych Neurol 2009;22(1):71.

74. Brayne C, Calloway P. The association of education and socioeconomic status with the Mini Mental State Examination and the clinical diagnosis of dementia in elderly people. Age \& Ageing 1990;19(2):91-6.

75. Launer LJ, Dinkgreve MA, Jonker C, Hooijer C, Lindeboom J. Are age and education independent correlates of the Mini-Mental State Exam performance of community-dwelling elderly? J Gerontol. 1993;48:271-7.

76. Prencipe M, Casini AR, Ferretti C, Lattanzio MT, Fiorelli M, Culasso F. Prevalence of dementia in an elderly rural population: Effects of age, sex, and education. J Neurol Neurosurg Psychiatry. 1996;60:628-33.

77. Katzman R. Education and the prevalence of dementia and Alzheimer's disease. Neurology. 1993;43:13-20.

78. Fratiglioni L, Grut M, Forsell Y, Viitanen M, Grafström M, Holmén K, et al. Prevalence of Alzheimer's disease and other dementias in an elderly urban population: relationship with age, sex, and education. Neurology. 1991;41:1886-92.

79. De Ronchi D, Fratiglioni L, Rucci P, Paternicò A, Graziani S, Dalmonte E. The effect of education on dementia occurrence in an Italian population with middle to high socioeconomic status. Neurology. 1998;50:1231-8.

80. Mortel KF, Meyer JS, Herod B, Thornby J. Education and occupation as risk factors for dementias of the Alzheimer and ischemic vascular types. Dementia. 1995;6:55-62.

81. Gatz M, Svedberg P, Pedersen NL, Mortimer JA, Berg S, Johansson B. Education and the risk of Alzheimer's disease: findings from the study of dementia in Swedish twins. J Gerontol B Psychol Sci Soc Sci. 2001;56:292-300.

82. Raiha I, Kaprio J, Koskenvuo M, Rajala T, Sourander L. Environmental differences in twin pairs discordant for Alzheimer's disease. J Neurol Neurosurg Psychiatry. 1998;65:785-7.

83. Stern Y, Gurland B, Tatemichi TK, Tang MX, Wilder D, Mayeux R. Influence of education and occupation on the incidence of Alzheimer's disease. JAMA. 1994;271:1004-10.

84. Evans DA, Hebert LE, Beckett LA, Scherr PA, Albert MS, Chown MJ, et al. Education and other measures of socioeconomic status and risk of incident Alzheimer disease in a defined population of older persons. Arch Neurol. 1997;54:1399-405.

85. Anstey K, Christensen H. Education, activity, health, blood pressure and apolipoprotein $\mathrm{E}$ as predictors of cognitive change in old age: A review. Gerontology. 2000;46:163-77. 
86. Glymour MM, Weuve J, Berkman LF, Kawachi I, Robins JM. When is baseline adjustment useful in analyses of change? An example with education and cognitive change. Am J Epidemiol. 2005;162:267-78.

87. Christensen H, Hofer SM, MacKinnon AJ, Korten AE, Jorm AF, Henderson AS. Age is no kinder to the better educated: absence of an association investigated using latent growth techniques in a community sample. Psychol Med. 2001;31:15-28.

88. Van Dijk KRA, Van Gerven PWM, Van Boxtel MPJ, Van der Elst W, Jolles J. No protective effects of education during normal cognitive aging: results from the 6-year follow-up of the Maastricht Aging Study. Psychol Aging. 2008;23:119.

89. Karlamangla AS, Miller-Martinez D, Aneshensel CS, Seeman TE, Wight RG, Chodosh J. Trajectories of cognitive function in late life in the United States: demographic and socioeconomic predictors. Am J Epidemiol. 2009;170:331-42.

90. Yu B, Ghosh P. Joint modeling for cognitive trajectory and risk of dementia in the presence of death. Biometrics. 2010;66:294-300.

91. Flynn JR. The mean IQ of Americans - Massive gains 1932 to 1978. Psychol Bull. 1984;95:29-51.

92. Kalantar-Zadeh K, Block G, Humphreys M, Kopple JD. Reverse epidemiology of cardiovascular risk factors in maintenance dialysis patients. Kidney Int. 2003;63:793-808.

93. Hernan MA, Alonso A, Logroscino G. Cigarette smoking and dementia: Potential selection bias in the elderly. Epidemiology. 2008;19:448-50.

94. Haneuse S, Schildcrout J, Crane P, Sonnen J, Breitner J, Larson E. Adjustment for selection bias in observational studies with application to the analysis of autopsy data. Neuroepidemiology. 2009;32:229-39.

95. Hernan MA, Hernandez-Diaz S, Robins JM. A structural approach to selection bias. Epidemiology. 2004;15:615-25.

96. Morgan SW, Christopher. Counterfactuals and causal inference: Methods and principles for social research. Cambridge (UK): Cambridge University Press; 2007.

97. Gabler N, Duan N, Liao D, Elmore JG, Ganiats TG, Kravitz RL. Dealing with heterogeneity of treatment effects: is the literature up to the challenge? Trials. 2009;10:43.

98. Kravitz RL, Duan N, Braslow J. Evidence-based medicine, heterogeneity of treatment effects, and the trouble with averages. Milbank Q. 2004;82:661-87.

99. Wang R, Lagakos SW, Ware JH, Hunter DJ, Drazen JM. Statistics in medicine - reporting of subgroup analyses in clinical trials. $\mathrm{N}$ Engl $\mathrm{J}$ Med. 2007;357:2189-94.

100. Terry MB, Wei Y, Esserman D. Maternal, birth, and early-life influences on adult body size in women. Am J Epidemiol. 2007;166:5-13.

101. Forrest CB, Riley AW. Childhood origins of adult health: A basis for lifecourse health policy. Health Aff. 2004;23:155-64.

102. Lu MK, M; Hogan, V; Jones, L; Wright, K; Halfon, N. Closing the BlackWhite gap in birth outcomes: A life-course approach. Ethn Dis. 2010;20(Suppl 2):62-72. 
103. Misra DP, Guyer B, Allston A. Integrated perinatal health framework: A multiple determinants model with a life span approach. Am J Prev Med.2003;25:65-75.

104. Wise PH. Framework as metaphor: The promise and peril of MCH life-course perspectives. Matern Child Health J 2003;7:151-6.

105. U.S. Department of Health and Human Services. Rethinking MCH: The life course model as an organizing framework. October 2010. USDHHS Health Resources and Services Adminsitration Maternal and Child Health Bureau.

106. Lemere C, Masliah E. Can Alzheimer disease be prevented by amyloidimmunotherapy? Nature Rev Neurol. 2010;6:108-19.

107. Carlson MC, Erickson KI, Kramer AF, Voss MW, Bolea N, Mielke M, et al. Evidence for neurocognitive plasticity in at-risk older adults: The experience corps program. J Gerontol A Biol Sci Med Sci. 2009;64:1275-82.

108. Kleim JA, Jones TA. Principles of experience-dependent neural plasticity: Implications for rehabilitation after brain damage. J Speech Lang Hear Res. 2008;51:S225-39.

109. Wolinsky FD, Unverzagt FW, Smith DM, Jones R, Stoddard A, Tennstedt SL. The ACTIVE cognitive training trial and health-related quality of life: Protection that lasts for 5 years. J Gerontol A Biol Sci Med Sci. 2006;61:1324-9. 